\title{
BMJ Open Cost-effectiveness analysis of renal replacement therapy strategies in Guangzhou city, southern China
}

\author{
Fei Yang (D) , ${ }^{1}$ Meixia Liao, ${ }^{2}$ Pusheng Wang, ${ }^{1}$ Yongguang Liu ${ }^{3}$
}

To cite: Yang F, Liao M, Wang $\mathrm{P}$, et al. Cost-effectiveness analysis of renal replacement therapy strategies in Guangzhou city, southern China. BMJ Open 2021;11:e039653. doi:10.1136/ bmjopen-2020-039653

\section{- Prepublication history and} additional materials for this paper is available online. To view these files, please visit the journal online (http://dx.doi org/10.1136/bmjopen-2020039653).

Received 22 April 2020 Revised 01 November 2020 Accepted 15 December 2020

Check for updates

(C) Author(s) (or their employer(s)) 2020. Re-use permitted under CC BY-NC. No commercial re-use. See rights and permissions. Published by BMJ.

${ }^{1}$ Tsinghua Shenzhen International Graduate School, Tsinghua University, Shenzhen, China

${ }^{2}$ Institute for Hospital Management, Tsinghua University, Shenzhen, China ${ }^{3}$ Organ Transplantation Center, Zhujiang Hospital, Guangzhou, China

\section{Correspondence to} Yongguang Liu; freeright@189.cn and Professor Pusheng Wang; wangps@tsinghua.edu.cn

\section{ABSTRACT}

Objectives This study aims to assess the costeffectiveness of three renal replacement therapy (RRT) modalities as well as proposed changes of scheduled policies in RRT composition in Guangzhou city.

Methods From a payer perspective, we designed Markov model-based cost-effectiveness analyses to compare the cost-effectiveness of three RRT modalities and four different scheduled policies to RRT modalities in Guangzhou over three time horizons (5, 10 and 15 years). The current situation (scenario 1: haemodialysis (HD), $73 \%$; peritoneal dialysis (PD), $14 \%$; kidney transplantation (TX), 13\%) was compared with three different scenarios: an increased proportion of incident RRT patients on PD (scenario 2: HD, 47\%; PD, 40\%; TX, 13\%); on TX (scenario 3: HD, 52\%; PD, 14\%; TX, 34\%); on both PD and TX (Scenario 4: HD, 26\%; PD, 40\%; TX, 34\%).

Results Over 5-year time horizon, HD was dominated by PD. At a willingness-to-pay (WTP) threshold of US $\$ 44$ 300 , TX was cost-effective compared with PD with an incremental cost-effectiveness ratio of US\$35518 per quality-adjusted life year (QALY) gained. The scenario 2 held a dominant position over the scenario 1, with a net saving of US\$ 5.92 million and an additional gain of 6.24 QALYs. The scenarios 3 and 4 were cost-effective compared with scenario 1 at a WTP threshold of US\$44300. The above results were consistent across the three time horizons.

Conclusions TX is the most cost-effective RRT modality, followed in order by PD and HD. The strategy with an increased proportion of incident patients on PD and TX is cost-effective compared with the current practice pattern at the given WTP threshold. The planning for RRT service delivery should incorporate efforts to increase the utilisation of PD and TX in China.

\section{INTRODUCTION}

Globally, the growing incidence and prevalence of end-stage renal disease (ESRD) is a major public health challenge and has resulted in a heavy economic burden on individuals and national healthcare systems. ${ }^{1}$ Patients with ESRD are treated with renal replacement therapy (RRT), which is available in three different modalities: haemodialysis (HD), peritoneal dialysis (PD) and kidney transplantation (TX). Currently, more than 2.5 million patients are receiving $\mathrm{RRT}^{2}$ with

\section{Strengths and limitations of this study}

To the author's knowledge, this is the first costeffectiveness analysis of three competing renal replacement therapy (RRT) modalities in end-stage renal disease patients in southern China.

- This is the only study that has explored the costeffectiveness of different scheduled policies to RRT modalities among incident patients from the payer perspective in China.

- Our study may potentially be biased by quoting some of transition probability parameters from studies of other countries where clinical practice might be different from China.

- The utility values derived from our own observational survey have the potential bias due to a relative small sample size and the difference in baseline characteristics of participants.

- Given the payer perspective of the analysis, this study did not consider indirect costs such as productivity loss and costs associated with caregivers.

the total ESRD expenditure accounting for $0.91 \%-7.1 \%$ of national health system expenditure among countries with high prevalence of ESRD. ${ }^{1}$ In China, there were approximately 1 million ESRD patients in 2017, but only $52 \%$ of them have received RRT. ${ }^{3}$ HD is by far the predominant RRT modality in mainland China, accounting for approximately $86 \%$ of dialysis patients. There is a large variation in PD uptake among different regions, ranging from $14 \%$ in mainland China to $73 \%$ in Hong Kong. ${ }^{4}$ It was estimated that the annual healthcare expenditure on dialysis in China is approximately US $\$ 50$ billion. $^{3}$ Each RRT modality is associated with different patterns of resource utilisation and health outcomes. In the context of ageing populations and global pandemics of chronic disease, the prevalence of ESRD is expected to rise by a greater extent in the future. Therefore, searching for a more cost-effective approach to clinical decision-making for ESRD is highly demanded. 
Multiple studies have been conducted to evaluate the cost-effectiveness of RRT modalities. However, most of the studies evaluated parts of RRT modalities, such as comparing different dialysis modalities with one another or a certain dialysis modality with TX..$^{5-7}$ Several studies have assessed the cost-effectiveness of all available RRT modalities, but most of them were conducted in developed countries, such as the UK, ${ }^{8}$ Japan, ${ }^{9}$ Denmark ${ }^{10}$ and Australia. ${ }^{11}$ The majority of these studies suggest that TX is the optimal RRT modality and PD is more favourable than HD from the viewpoint of cost-effectiveness. Nevertheless, HD is currently the predominant RRT modality in mainland China. Furthermore, several studies have assessed the cost-effectiveness with respect to changes in the composition of RRT modalities ${ }^{11-13}$ suggesting that increased provision of TX and PD contributes to reducing costs and increasing health outcomes. Few studies have forecasted the potential benefits through changing the composition of RRT modality in China. Therefore, this study aims to assess the cost-effectiveness of three RRT modalities as well as proposed changes in RRT composition in China.

\section{MATERIALS AND METHODS \\ Study design}

We conducted a Markov model-based cost-effectiveness analysis to compare the cost-effectiveness of three RRT modalities as first-line treatment strategies in Guangzhou. In addition, we constructed a dynamic population-based Markov model to evaluate the cost-effectiveness of three different scheduled policies to RRT modalities in comparison with the current practice in Guangzhou.

The following scenarios were considered.

Scenario 1: A model that represents the current distribution of RRT modality (HD, 73\%; PD, 14\%; TX, 13\%).

Scenario 2: A model with an increased proportion of incident RRT patients on PD at the expense of HD (HD, 47\%; PD, 40\%; TX, 13\%).

Scenario 3: A model with an increased proportion of incident RRT patients on TX at the expense of HD (HD, 52\%; PD, 14\%; TX, 34\%).

Scenario 4: A model with an increased proportion of incident RRT patients on PD and TX at the expense of HD (HD, 26\%; PD, 40\%; TX, 34\%).

The current distribution of RRT modality was based on data recorded by Chinese National Renal Data System (CNRDS) and Chinese Scientific Registry of Kidney Transplantation (CSRKT). The increased allocation of $40 \%$ of incident RRT patients to PD was based on the fact that PD accounted for approximately $40 \%$ of all RRTs in Hong Kong in 2016, and the increase in percentage distribution of TX was determined from the fact that the average percentage distribution of TX in 59 countries was approximately $34 \%$ in 2016, according to the US Renal Data System 2018 Annual Data Report. ${ }^{14}$

From a payer perspective, the assessment of costs was designed to include only direct medical costs for RRT

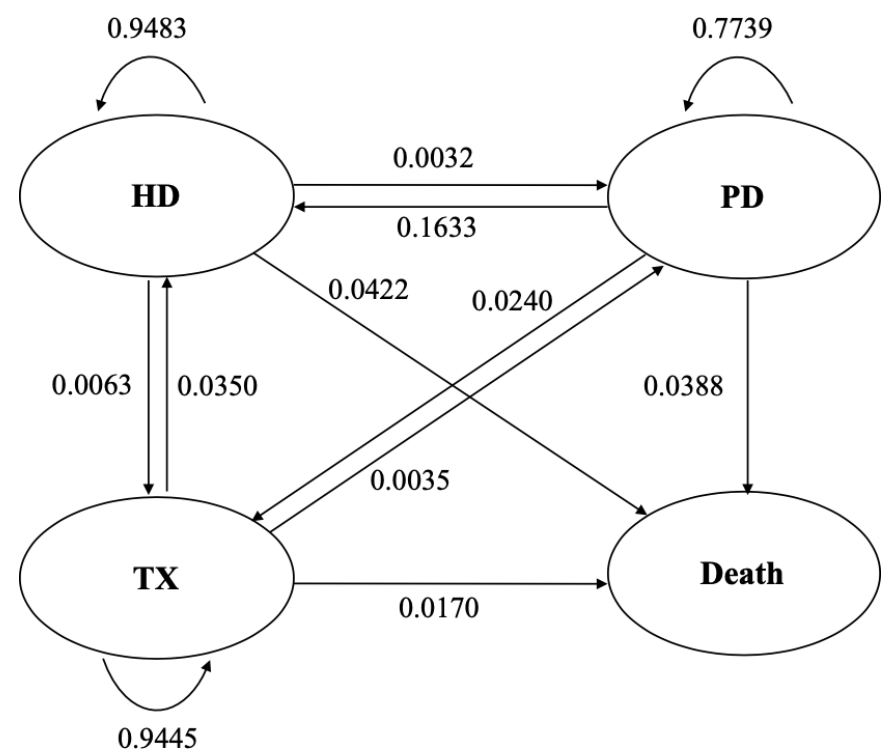

Figure 1 Schematic diagram of the Markov model. HD, haemodialysis;PD, peritoneal dialysis; TX, kidney transplantation

patients. The effectiveness is measured in terms of qualityadjusted life years (QALYs). The main outcome measures were total costs, total effectiveness expressed in terms of QALYs and incremental cost-effectiveness ratio (ICER) of competing strategies. The analyses were performed with three time horizons (5, 10 and 15 years).

\section{Model structure}

Markov models are frequently used to model the progression of chronic disease over time including ESRD. ${ }^{15}$ In this study, the ESRD Markov model was structured as a chain of four mutually exclusive states, including HD, PD, TX and death, as shown in figure 1 . All states could mutually transit to each other, except for the state of death which was absorbing state. Patients could stay at the same state or switch to a new state according to given transition probabilities for each 1-year cycle.

\section{Projection of the prevalence and incidence of RRT patients}

In the dynamic population-based Markov model for the scenario analysis, the future distribution of patients over the states depends on the initial prevalence, transition probabilities and the inflow of new patients. The initial prevalence and the inflow of new RRT patients for the model were estimated based mainly on data from CNRDS and CSRKT. The CNRDS is a nationwide, webbased, prospective renal data registration platform which collects structured data on dialysis patients in dialysis centres of China. ${ }^{16}$ The CSRKT is an official and national data acquisition system for TX in China. ${ }^{17}$

The initial prevalence for the model was derived from the RRT prevalence figures at the end of 2017 from CNRDS and CSRKT and was distributed among the RRT modalities based on the proportions observed at the end of 2017 in China: HD, 80\%; PD, 14\%; TX, 6\%. The expected inflow of new patients commencing RRT is 


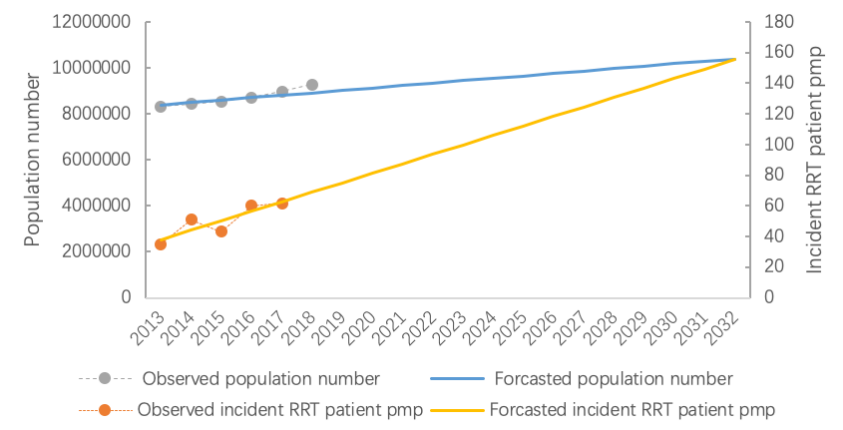

Figure 2 Forecasted population numbers and incident renal replacement therapy (RRT) patient per million population (ppm).

both dependent on the incidence of RRT and on demographic developments. The simple linear regression analyses with time as the independent variable were used to estimate the population numbers of Guangzhou and the inflow of new RRT patients per million population (pmp) over the period 2018-2032. The expected population numbers were derived from the total registered population of Guangzhou between 1985 and 2018 from Guangzhou Statistics Bureau. ${ }^{18}$ The expected inflow of new RRT patients pmp were extrapolated from the RRT incidence figures between 2012 and 2017 from CNRDS and CSRKT. Figure 2 shows the results from the forecasting models. The above extrapolated figures were used to further estimate the inflow of new RRT patients in Guangzhou over the period 2018-2032 for the scenario analysis.

\section{Health utilities}

We conducted a questionnaire survey on RRT patients in Zhujiang Hospital of Southern Medical University between August 2018 and October 2018. A total of 276 patients were included in this study through convenience sampling, with 112 patients for HD, 68 for PD and 97 for TX. The inclusion criteria for the study population were: ESRD patients treated with HD, PD or TX, age above 18 years, receiving the same RRT modality for at least 3 months, and physically and mentally capable of completing the survey with minimal assistance. Patients who had combination therapy of RRT modalities within 3 months were excluded from the study. All of the participants gave their written consent prior to their inclusion in the study. The study was approved by the Ethics Committee of Zhujiang Hospital of Southern Medical University. Table 1 shows the characteristics of the subjects.

The Kidney Disease Quality of Life Short Form (KDQOL-SFV.1.3) was used to measure health-related quality of life of RRT patients and generate the SF-6D health utility score. The KDQOL-SF is a self-reported questionnaire for assessing the quality of life of individuals with kidney disease and includes the RAND 36-Item Health Survey (SF-36) as the generic core. ${ }^{19}$ Using the methodology proposed by Brazier et $a t^{20} 11$ out of 36 items from SF-36 have been selected to derive SF-6D health utility scores. We further derived the utility weights for the determination of QALYs according to Chinese Hong Kong population-specific scoring algorithm. ${ }^{21}$ The SF-6D is a preference-based scoring system consisting of six dimensions: physical functioning, role limitations, social functioning, pain, mental health and vitality. ${ }^{20}$ The derived SF-6D health utility score has shown to be valid and reliable and been used to measure utility in Chinese patients on RRT. ${ }^{22} 23$

We also conducted a systematic review of full economic evaluations on RRT. $^{24}$ The review indicates that there is a lack of availability of utility data sources with high quality. As shown in table 2, the review shows that there is a wide range of utility values across the reviewed studies, with utility value of $\mathrm{HD}, \mathrm{PD}$ and TX ranging from 0.44 to $0.72,0.53$ to 0.81 and 0.57 to 0.9 , respectively. The reviewed studies that obtain utility values from their own observational surveys are likely to suffer from selection bias. Moreover, the reviewed studies that obtain utility values from previous literature fail to report the population used to derive utility values and it is difficult to define whether utility values are biased by the baseline characteristics of the individuals. Given that previous literature fails to provide utility data sources with high quality and our target population are ESRD patients in China, we choose to use the utility values derived from our own survey.

\section{Costs}

The annual direct medical cost of three RRT modalities was obtained from the study by Zhang et al. ${ }^{25}$ This retrospective observational cohort study obtained data from claims database of the Urban Employee Basic Medical Insurance and the Urban Resident Basic Medical Insurance of Guangzhou city from 2010 to 2013. The annual medical costs of HD and PD included costs for routine dialysis treatment and hospitalisations if needed. TX costs in the first year and subsequent years were assigned two different costs, with the first-year cost including extra costs of kidney transplant procedure. All costs were adjusted to 2018 US $\$$ value by using the gross domestic product (GDP) deflator index and purchasing power parity conversion rate from the International Monetary Fund World Economic Outlook Database. ${ }^{26}$

\section{Transition probabilities}

Transition probabilities were obtained from the best available published data. The transition probabilities of HD to $\mathrm{PD}, \mathrm{HD}$ to TX, HD to death and PD to death were derived from the CNRDS from 2011 to $2015 .{ }^{27}$ Other transition probabilities, including the transition probabilities of PD to HD, PD to TX, TX to HD, TX to PD and TX to death, were unavailable from Chinese database or studies and were obtained from the published studies based on clinical databases or populations of other countries. ${ }^{9} 1228$ Table 3 shows the parameter values and ranges used in the Markov model. 
Table 1 The characteristics of patients from Zhujiang Hospital of Southern Medical University that were used for the estimation of health utility

\begin{tabular}{|c|c|c|c|c|}
\hline Characteristics & $\begin{array}{l}\text { HD }(n=112) \\
n(\%)\end{array}$ & $\begin{array}{l}\text { PD (n=68) } \\
n(\%)\end{array}$ & $\begin{array}{l}\text { TX }(n=97) \\
n(\%)\end{array}$ & $P$ value \\
\hline Gender & & & & 0.877 \\
\hline Male & $55(49.11)$ & $35(51.47)$ & $51(52.58)$ & \\
\hline Female & $57(50.89)$ & $33(48.53)$ & $46(47.42)$ & \\
\hline Age $($ mean $\pm S D)$ & $52.71 \pm 15.26$ & $41.18 \pm 9.62$ & $41.84 \pm 11.00$ & $<0.001^{\star}$ \\
\hline Education & & & & $<0.001^{*}$ \\
\hline Primary school or below & $30(26.79)$ & $13(19.12)$ & $6(6.19)$ & \\
\hline Secondary school & $34(30.36)$ & $25(36.76)$ & $17(17.53)$ & \\
\hline High school & $28(25.00)$ & $15(22.06)$ & $30(30.93)$ & \\
\hline College or above & $20(17.86)$ & $15(22.06)$ & $44(45.36)$ & \\
\hline Annual income level (CNY) & & & & $0.001^{*}$ \\
\hline$<30000$ & $46(41.07)$ & $44(64.71)$ & $33(34.02)$ & \\
\hline $30000-120000$ & $44(39.29)$ & $16(23.53)$ & $33(34.02)$ & \\
\hline$>120000$ & $22(19.64)$ & $8(11.76)$ & $31(31.96)$ & \\
\hline Marital status & & & & 0.646 \\
\hline Not married & 15 (13.39) & $12(17.65)$ & $17(17.53)$ & \\
\hline Married & 97 (86.61) & $56(82.35)$ & $80(82.47)$ & \\
\hline Employment & & & & $<0.001^{*}$ \\
\hline Employed & $27(24.11)$ & $22(32.35)$ & $47(48.45)$ & \\
\hline Unemployed & $37(33.04)$ & $41(60.29)$ & $41(42.27)$ & \\
\hline Retired & $48(42.86)$ & $5(7.35)$ & $9(9.28)$ & \\
\hline Insurance & & & & 0.010 \\
\hline UEBMI & $64(57.14)$ & $38(55.88)$ & $46(47.42)$ & \\
\hline URBMI & 29 (25.89) & 7 (10.29) & 29 (29.90) & \\
\hline Others & 19 (16.96) & 23 (33.82) & 22 (22.68) & \\
\hline
\end{tabular}

Continuous variables were analysed by one-way analysis of variance.

Categorical variables were analysed by Pearson $\chi^{2}$ test.

${ }^{*} \mathrm{p}<0.05$.

HD, haemodialysis; PD, peritoneal dialysis; TX, kidney transplantation; UEBMI, Urban Employee Basic Medical Insurance; URBMI, Urban Resident Basic Medical Insurance.

\section{Model analysis}

To compare the cost-effectiveness of three RRT modalities, the decision analytic model was constructed as a decision tree with three similar Markov models as shown in online supplemental figure 1 . To assess the cost-effectiveness of proposed changes in RRT modality utilisation versus current practice, the decision analytic model was constructed as a decision tree with four dynamic population-based Markov models as shown in online supplemental figure 2. In the dynamic Markov models, for each 1-year cycle, new patients were added to an initial state from where they are assigned to HD, PD or TX based on the proportions given in scenarios. The simulation was set to start at the beginning of 2018 . The models were run for 5,10 and 15 cycles, respectively. We used the half-cycle correction and discounted both costs and health outcomes at a common $5 \%$ rate. $^{29}$ The ICER less than one time of GDP per capita or between one and three times of GDP per capita was considered cost-effective and very cost-effective, respectively. ${ }^{30}$ Using the corresponding 2018 GDP per capita of Guangzhou, ${ }^{31}$ the thresholds of willingness-to-pay (WTP) for being very cost-effective and cost-effective in this study were US\$44300 and US\$132 900, respectively.

\section{Sensitivity analysis}

Both one-way sensitivity analyses and probabilistic sensitivity analysis (PSA) were conducted to test the uncertainty and robustness of the results to changes in model parameters. For one-way sensitivity analyses, 95\% CIs of model parameters were used. The following parameters were analysed: utilities, costs and transition probabilities except those whose 95\% CIs were not reported in the literature. Overall results of one-way sensitivity analyses are presented in the form of a tornado diagram. The PSA took into account the uncertainties of utility parameters 
Table 2 Health utility values used in economic evaluations of RRT

\begin{tabular}{|c|c|c|c|c|}
\hline Modality & HD & PD & TX & \\
\hline \multicolumn{5}{|l|}{ Reference } \\
\hline Moradpour et a/ ${ }^{43}$ & 0.72 & 0.75 & 0.82 & \\
\hline Rosselli et al $^{44}$ & 0.576 & 0.668 & 0.796 & \\
\hline Jensen et $a l^{45}$ & 0.44 & 0.65 & 0.86 & \\
\hline Shimizu et $a l^{9}$ & 0.44 & 0.53 & LT: 0.71 & DT: 0.57 \\
\hline Villa et al ${ }^{12}$ & 0.69 & 0.69 & 0.81 & \\
\hline Haller et $\mathrm{al}^{13}$ & 0.66 & 0.81 & 0.9 & \\
\hline Howard et al ${ }^{11}$ & 0.55 & 0.55 & $\begin{array}{l}\text { First-year } \\
\text { TX utility } \\
0.73\end{array}$ & $\begin{array}{l}\text { Second- } \\
\text { year TX } \\
\text { utility } 0.70\end{array}$ \\
\hline $\begin{array}{l}\text { Kontodimopoulos } \\
\text { et } a l^{46}\end{array}$ & 0.639 & 0.599 & 0.716 & \\
\hline de Wit et al ${ }^{47}$ & 0.66 & 0.71 & 0.9 & \\
\hline Average & 0.60 & 0.66 & 0.77 & \\
\hline This study & 0.61 & 0.61 & 0.73 & \\
\hline
\end{tabular}

DT, deceased-donor transplantation; HD, haemodialysis; LT, living-donor transplantation; PD, peritoneal dialysis; RRT, renal replacement therapy; TX, renal transplantation.

by using applied distributions. As recommended by Briggs et $a l^{32}$ utility parameters were assigned a beta distribution. The PSA used a Monte Carlo simulation with 10000 iterations. The results of PSA are presented in the form of a cost-effectiveness acceptability curve. We also performed a sensitivity analysis for policy parameters, in which we repeated the calculations of Markov model with a range of values for scheduled proportion of HD, PD and TX, and compared the resulting costs and effectiveness to the current policy.

We used the Stata Statistical Software V.14.1 (StataCorp LLC, College Station, Texas, USA) and TreeAge Pro 2020 R1.1 software programme (TreeAge Software, Williamstown, Massachusetts, USA) for statistical analyses.

\section{Patient and public involvement}

This study was conducted without patient or public involvement.

\section{RESULTS}

\section{Cost-effectiveness analysis of three RRT modalities}

Table 4 shows the results of cost-effectiveness analysis of three RRT modalities over three time horizons. Focusing on the results of 5-year time horizon, HD was dominated by $\mathrm{PD}$ as it yielded both higher cost (US $\$ 115730$ vs US\$106 194) and lower effectiveness (2.46 QALYs vs 2.49 QALYs). TX had the highest effectiveness (3.06 QALYs) but with higher cost than PD (US\$126351 vs US\$106 194). The ICER was US $\$ 35518$ per QALY gained for TX over PD and was lower than the WTP threshold of one time of GDP per capita (US $\$ 44300$ ). For the analyses over 10-year and 15-year time horizons, the results were consistent with that of 5 -year time horizon.

\section{Cost-effectiveness analysis of four scenarios}

Table 5 shows the results of cost-effectiveness analysis of four scenarios over three time horizons. Over the 5-year time horizon, the total discounted costs and QALYs for the base composition of RRT modality (scenario 1) were US\$675.30 million and 14648.48 QALYs, respectively. The scenario 2 held a dominant position over the scenario 1, with lower costs and higher effectiveness. An increase in PD to $40 \%$ of incident RRT patient at the expense of HD (scenario 2) resulted in a net saving of US\$5.92 million and a small increase in total QALYs of 6.24. The marginally higher value for QALY at marginally higher cost translated into an ICER of US\$38452 per QALY gained for scenario 3 and US\$7786 per QALY gained for scenario 4 in comparison to scenario 1 , both of which were lower than the WTP threshold of one time of GDP per capita (US\$44 300). Results over longer time horizons of 10 and 15 years were consistent with the 5 -year results.

\section{Sensitivity analysis}

Figure 3 shows the tornado diagrams for three RRT modalities' analyses over 5-year time horizon. The top three most influential parameters were the utilities of TX and HD and costs of HD. Of note, there was only one cost-effectiveness threshold identified in the tornado diagrams, which indicates that all parameter changes could not change the optimal strategy. As can be seen from the $\mathrm{x}$-axis, the net benefit never falls below zero, indicating that TX remained cost-effective relative to HD and PD across the uncertainty range of each parameter at a WTP threshold of US\$132900 per QALY. Figure 4 shows the cost-effectiveness acceptability curve of three RRT modalities over 5-year time horizon at different values of WTP thresholds. Using an WTP threshold of US\$ 132900 per QALY, the probabilities of HD, PD and TX being the optimal treatment strategy were $12.95 \%$, $15.05 \%$ and $72.00 \%$, respectively (online supplemental figure 3 ). Figure 5 shows the sensitivity analysis for policy parameters. The gains in QALYs increase in the proportion of TX and costs savings increase in the proportion of PD. The results of sensitivity analyses were consistent across the three time horizons considered.

\section{DISCUSSION}

From a payer perspective, this study conducted a Markov model-based cost-effectiveness analysis to compare the cost-effectiveness of three RRT modalities and four different scheduled policies to RRT modalities in Guangzhou. The results suggest TX is the most cost-effective RRT modality, followed in order by PD and HD. Assigning an increased proportion of incident RRT patients on PD and TX would result in either a dominant position over the current policy predominated by HD or an ICER well below the given WTP threshold. Results were consistent 
Table 3 Parameter values and ranges used in the Markov model

\begin{tabular}{|c|c|c|c|c|}
\hline \multirow[b]{2}{*}{ Parameter } & \multirow[b]{2}{*}{ Value (mean) } & \multicolumn{2}{|c|}{ Range (95\% Cl) } & \multirow[b]{2}{*}{ Reference } \\
\hline & & Lower & Upper & \\
\hline \multicolumn{5}{|l|}{ Health utility } \\
\hline PD & 0.61 & 0.58 & 0.64 & \\
\hline $\mathrm{TX}$ & 0.73 & 0.71 & 0.75 & \\
\hline PD & 24547 & 23175 & 25986 & \\
\hline \multicolumn{5}{|l|}{$\mathrm{TX}$} \\
\hline Initial year & 40196 & 34652 & 46763 & \\
\hline Subsequent year & 28313 & 18577 & 30998 & \\
\hline HD to $T X$ & 0.0063 & 0.0048 & 0.0081 & \\
\hline HD to death & 0.0422 & 0.0340 & 0.0500 & \\
\hline PD to PD & 0.7739 & & & \\
\hline PD to HD & 0.1633 & & & Shimizu et $a l^{9}$ \\
\hline PD to TX & 0.0240 & & & Yang et $a l^{28}$ \\
\hline PD to death & 0.0388 & 0.0170 & 0.0610 & 2012-2015 CNRDS ${ }^{27}$ \\
\hline TX to TX & 0.9445 & & & Villa et $a l^{12}$ \\
\hline TX to HD & 0.0350 & & & \\
\hline
\end{tabular}

CNRDS, Chinese National Renal Data System; HD, haemodialysis; PD, peritoneal dialysis; TX, kidney transplantation.

Table 4 Cost-effectiveness analysis of three renal replacement therapy modalities over three time horizons

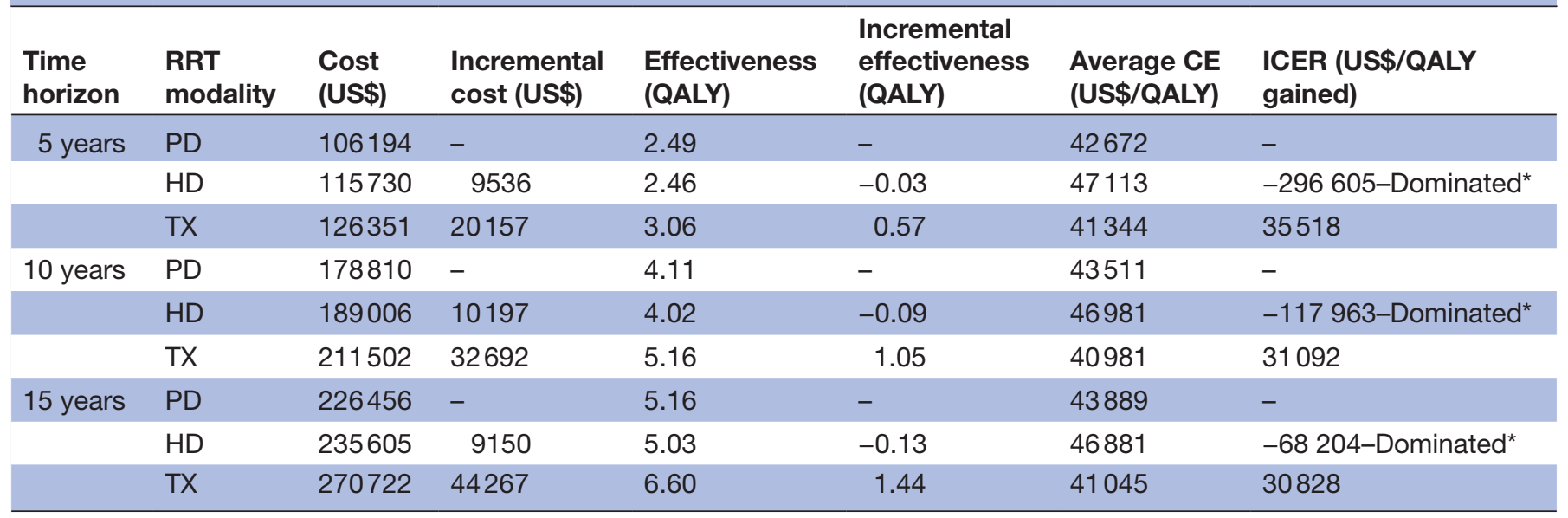

*Dominated: more costly and less effective.

HD, haemodialysis; ICER, incremental cost-effectiveness ratio; PD, peritoneal dialysis; QALY, quality-adjusted life years; RRT, renal replacement therapy; TX, kidney transplantation. 


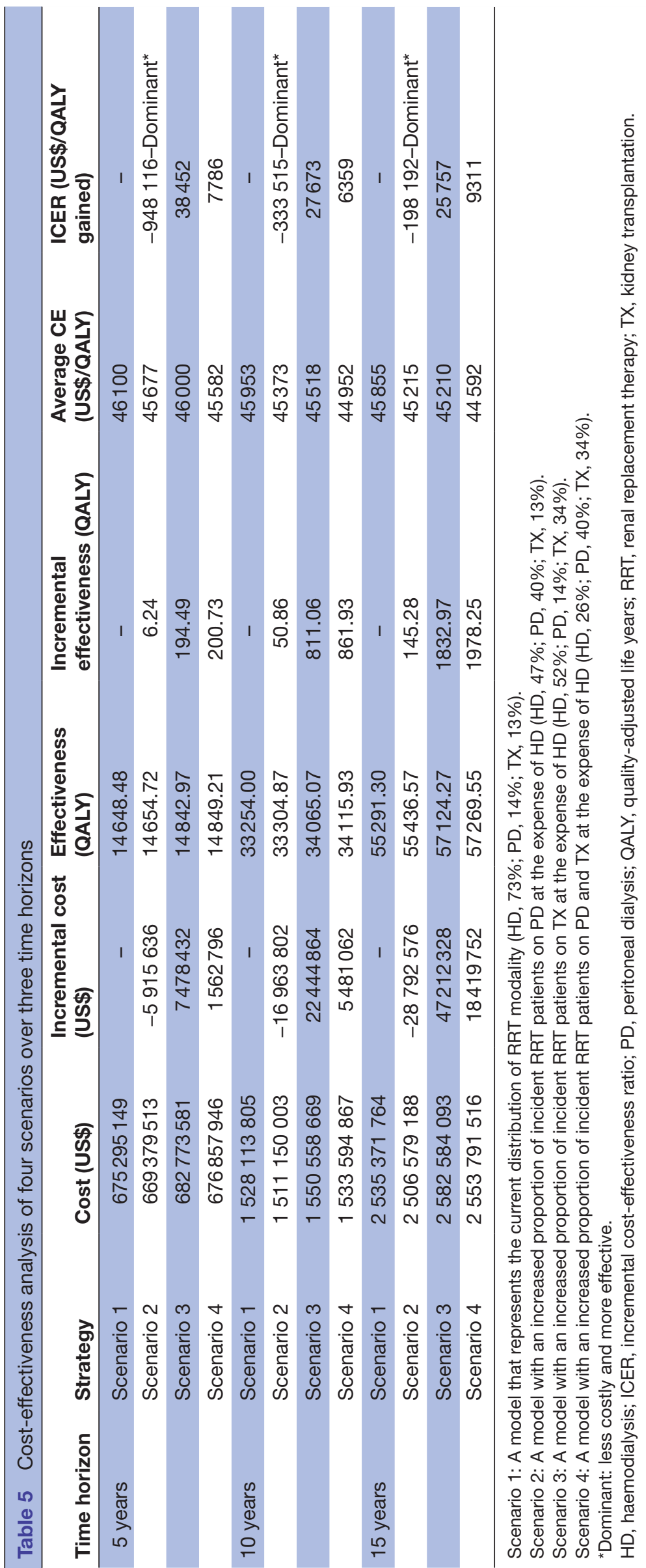


Tornado Diagram - Net Monetary Benefits (WTP: 132,900.00)

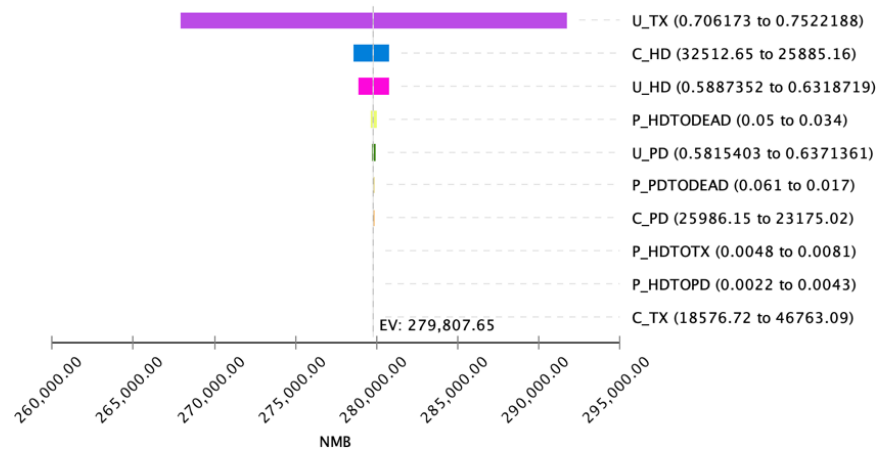

Figure 3 The tornado diagram for three renal replacement therapy modalities analysis over 5-year time horizon.

over three time horizons of 5,10 and 15 years. Our findings concur with previous studies on this topic conducted in other developed countries, such as Japan, ${ }^{9}$ Australia, ${ }^{11}$ Spain $^{12}$ and Austria. ${ }^{13}$

Our study suggests that PD is dominant over HD and the utilisation shift from HD to PD is associated with reduced costs and modest improvements in health outcomes, which is consistent with previous studies. ${ }^{5628}$ Due to its lower cost and less requirement for trained medical staff and technical support, a number of countries have established PD-First or PD-Favored policies to recommend $\mathrm{PD}$ as a preferred dialysis modality. ${ }^{3}$ The PD-First policy has made Hong Kong the region with the highest utilisation of PD and with a 73\% of PD uptake among dialysis patients in 2016. ${ }^{4}$ Nevertheless, HD is by far the predominant RRT modality in mainland China, accounting for approximately $86 \%$ of dialysis patients. Study has shown that home HD has similar cost-effectiveness to PD in many settings, ${ }^{33}$ but we considered only centre HD in our sample because of the limited utilisation of home HD in China. Actually, Renji Hospital Affiliated to Shanghai Jiao Tong University School of Medicine successfully carried out the first home HD treatment in mainland China on 27 April 2020, which was regarded as a milestone of the

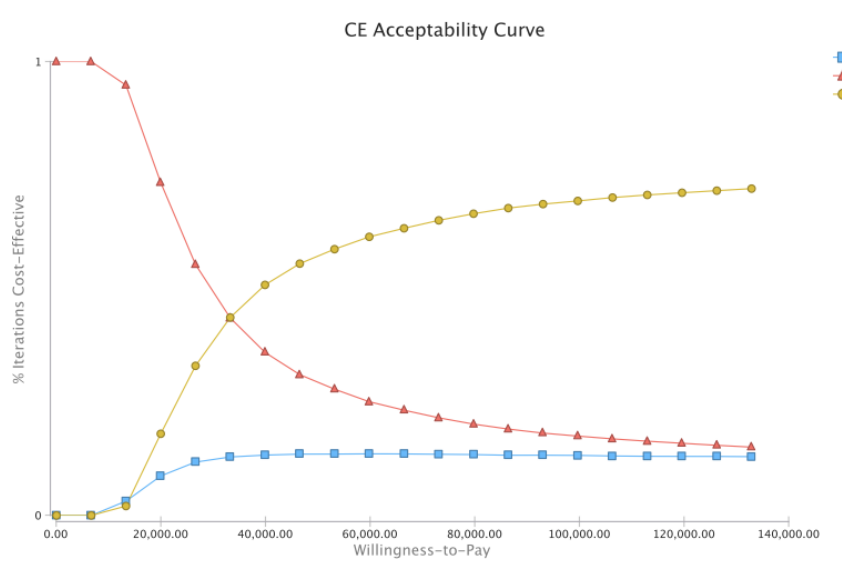

Figure 4 The cost-effectiveness acceptability curve of three renal replacement therapy modalities over 5-year time horizon.

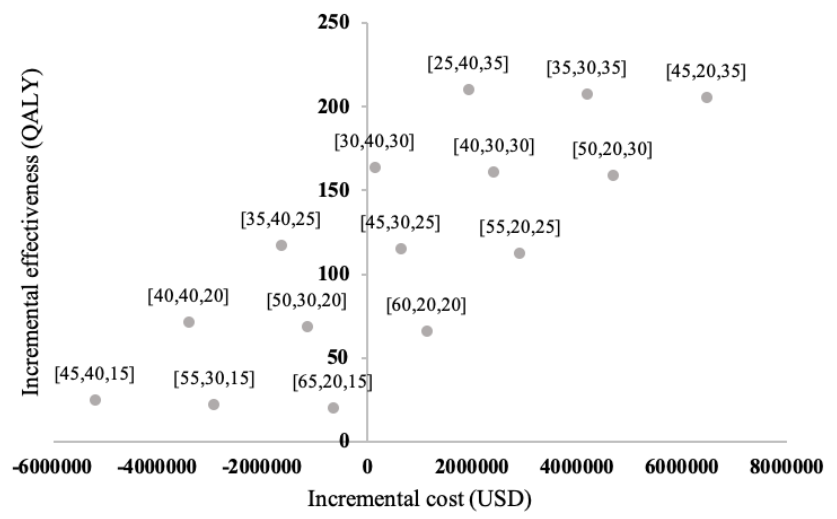

Figure 5 Incremental cost (US\$) and incremental effectiveness (quality-adjusted life year (QALY)) over 5 year time horizon for different scheduled policies compared with current scheduled policy.

zero breakthrough in home HD. ${ }^{34}$ The low utilisation of PD may be partially attributed to the different financial incentives caused by Zero-Markup Drug Policy which removes the mark-up for sale of medicines in hospitals. ${ }^{35}$ The costs of dialysis fluids for PD are categorised as medication costs which occupy approximately $86 \%$ of its total medical costs. ${ }^{25}$ In contrast, medication costs account for only approximately $20 \%$ of total HD costs, and most of HD costs are categorised as non-medication treatment costs. The differences in cost structures provide few financial incentives for doctors and hospitals to prescribe PD. Moreover, the relatively low marginal cost of adding a new HD patient stimulates providers to maximise the use of their HD units. ${ }^{36}$ Other patient-related factors could also further hinder the use of PD, including inadequate patient information, non-standard operation and socioeconomic disadvantages such as living in less favourable environment. ${ }^{35} 37$

Although it has been shown consistently across countries that TX is the optimal RRT modality, the utilisation of TX as the first-line treatment modality is primarily limited by the availability of donor organs. In 2017, only 10793 patients received TX with 30502 patients on the waiting list, indicating a considerable donor-organ shortfall in China. ${ }^{17}$ Living-donor transplants account for $64 \%$ of kidney transplants and there is no typical brain death donor as it opposes Chinese religious beliefs. ${ }^{38}$ The lack of correct attitude towards organ donation among the general public may constitute a barrier to increasing the donation rate, such as the ideas of maintaining the integrity of the body and misleading opinions of organ buying and selling. ${ }^{39}$ Moreover, the inactive involvement of medical workers in raising organ donor awareness and recruiting donors hinders the availability of donor organs. Therefore, it is recommended to implement specific strategies aimed at increasing kidney donations, such as increasing the use of expanded criteria donors and improving donor transplant coordination. In addition, study shows that Transplant Procurement 
Management (TPM) training programmes have positive perceived effects on professional competence development and career evolutions of healthcare workers in organ donation and transplantation. ${ }^{40}$ Launching TPM in more hospitals in China may keep health professionals active in organ donation and transplantation and further contribute to increase in organ donation figures.

Our findings provide evidence that increased allocation of incident RRT patients to PD and TX are likely to bringing about a more efficient resource distribution invested to treat ESRD. The planning for RRT service delivery should incorporate efforts to increase the utilisation of PD and TX, especially in the context of high prevalence of ESRD and limited healthcare resources in China. From a payer perspective, there is a need to revise the payment mechanism in a way to encourage valuebased healthcare decisions. Several countries have developed specific models aimed at facilitating value-based integrated renal care for patients, such as ESRD Treatment Choices in $\mathrm{USA}^{41}$ and the Ontario Renal Network in Canada. ${ }^{42}$

To the authors' knowledge, this is the first costeffectiveness analysis of three competing RRT modalities in ESRD patients in southern China. Moreover, this is the only study that has explored the cost-effectiveness of different scheduled policies to RRT modalities among incident patients from the payer perspective in China. Efforts were also made to collect first-hand data on health utilities to reflecting the actual health gains of Chinese ESRD patients commencing RRT modalities. Our findings may contribute to the better decision-making about the cost-effective ESRD management for the general public, nephrologists and health policymakers.

A number of limitations should be considered in this study. First, our study may potentially be biased by quoting some of transition probability parameters from studies of other countries where clinical practice might be different from China. Future research with more comprehensive China-based clinical and epidemiological data will facilitate economic evaluations of ESRD. Second, we assumed that the linear trends in the forecasting models were maintained over three time horizons, but these trends might be influenced by various factors, which could undermine the predictive validity in long term. For example, the growth rate of RRT incidence might be changed by the ESRD intervention strategies implemented in the future. Therefore, the forecasted data in long term could only be used for reference. Third, the utility values derived from our own observational survey have the potential bias due to a relative small sample size and the difference in baseline characteristics of participants. Finally, given the payer perspective of the analysis, this study did not consider indirect costs such as productivity loss and costs associated with caregivers. Future studies from a societal perspective would further substantiate the cost-effectiveness of RRT modalities.

In conclusion, this study indicates that TX and PD are more favourable than $\mathrm{HD}$, and the strategy with an increased proportion of incident patients on PD and TX is cost-effective compared with the policy currently being followed at the given WTP threshold. The planning for RRT service delivery should incorporate efforts to increase the utilisation of PD and TX in the future.

\section{Twitter Fei Yang @Summer2020Summer}

Contributors FY designed the study and performed the questionnaire survey. YL provided the data. ML and FY analysed the data, drafted and revised the manuscript. PW and YL provided critical review, advice and consultation throughout the writing of the manuscript. All authors approved the final version of the manuscript.

Funding This study was funded by 2018 Ministry of Education Humanities and Social Sciences General Project 'Technical Sociology Research on Chinese Organ Donation from a Multidimensional Perspective' (18YJA840011), Clinical Cultivation Project Foundation of Southern Medical University (LC2016PY029) and Shenzhen Key Research Base of Humanities and Social Sciences (N/A).

Competing interests None declared.

Patient consent for publication Not required.

Ethics approval This study did not involve clinical data from interventional therapies, clinical drug trials and private data like patient ID and name. Ethics Committee of Zhujiang Hospital of Southern Medical University granted ethical approval for the questionnaire survey on health related quality of life in ESRD patients through quick review channel without an approval ID. Written consent was obtained from each participant after explaining the study, its objective, benefits and its importance. Information were recorded anonymously and confidentiality and beneficence were assured throughout the study period.

Provenance and peer review Not commissioned; externally peer reviewed.

Data availability statement Data are available upon reasonable request. Data may be obtained from a third party and are not publicly available. The data from Chinese National Renal Data System (CNRDS) and Chinese Scientific Registry of Kidney Transplantation (CSRKT) may be obtained from a third party and are not publicly available. Other data are available upon reasonable request. Write to Fei Yang through daodanyydy163@163.com to place a reasonable request for the anonymised version and transcripts of the qualitative data.

Supplemental material This content has been supplied by the author(s). It has not been vetted by BMJ Publishing Group Limited (BMJ) and may not have been peer-reviewed. Any opinions or recommendations discussed are solely those of the author(s) and are not endorsed by BMJ. BMJ disclaims all liability and responsibility arising from any reliance placed on the content. Where the content includes any translated material, BMJ does not warrant the accuracy and reliability of the translations (including but not limited to local regulations, clinical guidelines, terminology, drug names and drug dosages), and is not responsible for any error and/or omissions arising from translation and adaptation or otherwise.

Open access This is an open access article distributed in accordance with the Creative Commons Attribution Non Commercial (CC BY-NC 4.0) license, which permits others to distribute, remix, adapt, build upon this work non-commercially, and license their derivative works on different terms, provided the original work is properly cited, appropriate credit is given, any changes made indicated, and the use is non-commercial. See: http://creativecommons.org/licenses/by-nc/4.0/.

ORCID iD

Fei Yang http://orcid.org/0000-0001-8234-5616

\section{REFERENCES}

1 Ismail $\mathrm{H}$, Abdul Manaf MR, Abdul Gafor AH, et al. International comparisons of economic burden of end-stage renal disease to the National Healthcare Systems. IIUM Med J Malaysia 2019;18:188-96.

2 Bikbov B, Purcell CA, Levey AS, et al. Global, regional, and national burden of chronic kidney disease, 1990-2017: a systematic analysis for the Global Burden of Disease Study 2017. Lancet 2020;395:709-33.

3 Yang C-W, Harris DCH, Luyckx VA, et al. Global case studies for chronic kidney disease/end-stage kidney disease care. Kidney Int Supp/ 2020;10:e24-48. 
4 Li PK-T, Chow KM, Van de Luijtgaarden MWM, et al. Changes in the worldwide epidemiology of peritoneal dialysis. Nat Rev Nephrol 2017;13:90-103.

5 Treharne C, Liu FX, Arici M, et al. Peritoneal dialysis and in-centre haemodialysis: a cost-utility analysis from a UK payer perspective. Appl Health Econ Health Policy 2014;12:409-20.

6 Wong $\mathrm{CKH}$, Chen J, Fung SKS, et al. Lifetime cost-effectiveness analysis of first-line dialysis modalities for patients with end-stage renal disease under peritoneal dialysis first policy. BMC Nephrol 2020;21:42

7 Bongiovanni I, Couillerot-Peyrondet AL, Sambuc C. Costeffectiveness analysis of various strategies of end-stage renal disease patients' care in France. Nephrol Ther 2016;12:104-15.

8 Nagaraja P, Lodhi V, Garrell D, et al. SP682A cost and outcomes analysis of renal replacement therapies using real-world quality of life data. Nephrol Dial Transplant 2015;30:iii604.

9 Shimizu U, Saito S, Lings Y, et al. Cost-effectiveness achieved through changing the composition of renal replacement therapy in Japan. J Med Econ 2012;15:444-53.

10 Jensen CE, Sorensen P, Petersen KD. In Denmark kidney transplantation is more cost-effective than dialysis. Dan Med $J$ 2014;61:A4796.

11 Howard K, Salkeld G, White S, et al. The cost-effectiveness of increasing kidney transplantation and home-based dialysis. Nephrology 2009;14:123-32.

12 Villa G, Fernández-Ortiz L, Cuervo J, et al. Cost-effectiveness analysis of the Spanish renal replacement therapy program. Perit Dial Int 2012;32:192-9.

13 Haller M, Gutjahr G, Kramar R, et al. Cost-effectiveness analysis of renal replacement therapy in Austria. Nephrology Dialysis Transplantation 2011;26:2988-95.

14 Saran R, Robinson B, Abbott KC, et al. Us renal data system 2018 annual data report: epidemiology of kidney disease in the United States. Am J Kidney Dis 2019;73:A7-8.

15 Briggs A, Sculpher M. An introduction to Markov modelling for economic evaluation. Pharmacoeconomics 1998:13:397-409.

16 Xie F, Zhang D, Wu J, et al. Design and implementation of the first nationwide, web-based Chinese Renal Data System (CNRDS). BMC Med Inform Decis Mak 2012;12:11.

17 National Health Commission of the People's Republic of China. National Medical Service and Quality Safety Report 2018. scientific and technological documentation press, Beijing, 2019.

18 Guangzhou Statistics Bureau: Guangzhou Statistical Yearbook 2019. Available: http://210.72.4.58/SRMC/yearBooks/2019/directory/ content.html?02-03 [Accessed 23 Mar 2020].

19 Hays RD, Kallich JD, Mapes DL. Kidney Disease Quality of Life Short Form (KDQOL-SFTM), version 1.3: a manual for use and scoring. Washington, DC: RAND, 1997. https://www.rand.org/content/dam/ rand/pubs/papers/2006/P7994.pdf

20 Brazier J, Roberts J, Deverill M. The estimation of a preferencebased measure of health from the SF-36. J Health Econ 2002;21:271-92.

21 McGhee SM, Brazier J, CLK L. Quality-adjusted life years: population-specific measurement of the quality component. Hong Kong Medical Journal 2011;17:17-21.

22 Wong CKH, Chen JY, Fung SKS, et al. Health-related quality of life and health utility of Chinese patients undergoing nocturnal home haemodialysis in comparison with other modes of dialysis. Nephrology 2019;24:630-7.

23 Pan C-W, Wu Y, Zhou H-J, et al. Health-related quality of life and its factors of hemodialysis patients in Suzhou, China. Blood Purif 2018;45:327-33

24 Yang F, Liao M, Wang P, et al. The cost-effectiveness of kidney replacement therapy modalities: a systematic review of full economic evaluations. Appl Health Econ Health Policy 2020;395

25 Zhang H, Zhang C, Zhu S, et al. Direct medical costs of end-stage kidney disease and renal replacement therapy: a cohort study in Guangzhou City, southern China. BMC Health Serv Res 2020;20:122.
26 CCEMG-EPPI-Centre Cost Converter. Available: http://eppi.ioe.ac. uk/costconversion/ default.aspx [Accessed 21 Mar 2020].

27 Liu J, Hutton DW, Gu Y, et al. Financial implications of dialysis modalities in the developing world: a Chinese perspective. Perit Dial Int 2020;40:193-201.

28 Yang F, Lau T, Luo N. Cost-effectiveness of haemodialysis and peritoneal dialysis for patients with end-stage renal disease in Singapore. Nephrology 2016;21:669-77.

29 Gold MR, Siegel JE, Russell LB, et al. Cost-effectiveness in health and medicine. New York: Oxford University Press, 1996.

30 Robinson LA, Hammitt JK, Chang AY, et al. Understanding and improving the one and three times GDP per capita cost-effectiveness thresholds. Health Policy Plan 2017;32:141-5.

31 Guangzhou Statistics Bureau: Guangzhou Statistical Yearbook 2019. Available: http://210.72.4.58/portal/querylnfo/statisticsYearbook/ index [Accessed 23 Mar 2020].

32 Briggs A, Claxton K, Sculpher M. Decision modelling for health economic evaluation. In: Gray A, Briggs A, eds. Making decision models probalistic. 1st edn. New York: Oxford University Press Inc, 2006: 77-118.

33 Winkelmayer WC, Weinstein MC, Mittleman MA, et al. Health economic evaluations: the special case of end-stage renal disease treatment. Med Decis Making 2002;22:417-30.

34 Renji Hospital affiliated to Shanghai Jiaotong University school of medicine successfully implemented the first home hemodialysis treatment in mainland China. J Shanghai Jiaotong University 2020;40:678

35 Zhou L, Zeng X-X, Fu P. Community hemodialysis in China: opportunities and challenges. Chin Med J 2017;130:2143-6.

36 Liu FX, Gao X, Inglese G, et al. A global overview of the impact of peritoneal dialysis first or favored policies: an opinion. Perit Dial Int 2015;35:406-20.

37 Walker RC, Howard K, Tong A, et al. The economic considerations of patients and caregivers in choice of dialysis modality. Hemodial Int 2016;20:634-42.

38 Viklicky O. First registry report on kidney transplantation in China. Transpl Int 2020;33:713-4.

39 Istrate MG, Harrison TR, Valero R, et al. Benefits of transplant procurement management (TPM) specialized training on professional competence development and career evolutions of health care workers in organ donation and transplantation. Exp Clin Transplant 2015:13:148-55.

40 Ming Y, Tu B, Zhuang Q. Current situation of organ donation in China. In: Tsoulfas G, ed. Organ donation and transplantation: current status and future challenges. 1st edn. London, UK: I ntechOpen, 2018. https://www.intechopen.com/books/organ-donation-andtransplantation-current-status-and-future-challenges/currentsituation-of-organ-donation-in-china

41 ESRD Treatment Choices (ETC) Model. Centers for medicare \& medicaid services. Available: https://innovation.cms.gov/innovationmodels/esrd-treatment-choices-model [Accessed 28 Mar 2020].

42 Gilardino RE, González-Pier E, Brabata C. End-Stage renal disease models in the Americas: optimizing resources to achieve better health outcomes. Value Health Reg Issues 2018;17:115-8.

43 Moradpour A, Hadian M, Tavakkoli M. Economic evaluation of end stage renal disease treatments in Iran. Clin Epidemiol Glob Health 2020;8:199-204.

44 Rosselli D, Rueda J-D, Diaz C. Cost-effectiveness of kidney transplantation compared with chronic dialysis in end-stage renal disease. Saudi J Kidney Dis Transp/ 2015;26:733-8.

45 Jensen CE, Sørensen P, Petersen KD. In Denmark kidney transplantation is more cost-effective than dialysis. Dan Med $J$ 2014;61:A4796.

46 Kontodimopoulos N, Niakas D. An estimate of lifelong costs and QALYs in renal replacement therapy based on patients' life expectancy. Health Policy 2008;86:85-96.

47 de Wit GA, Ramsteijn PG, de Charro FT. Economic evaluation of end stage renal disease treatment. Health Policy 1998;44:215-32. 